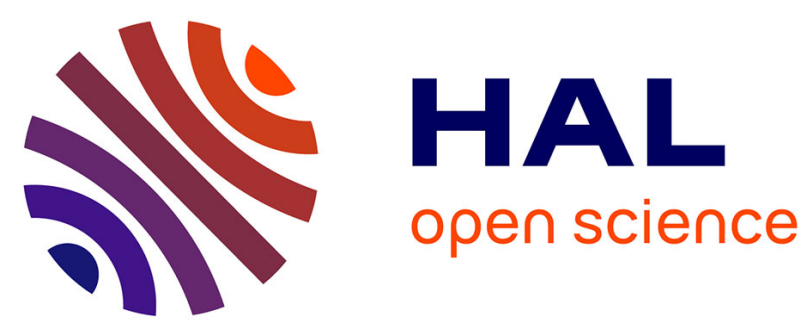

\title{
ROCK: A Beamline Tailored for Catalysis and Energy-Related Materials from ms Time Resolution to $\mu \mathrm{m}$ Spatial Resolution
}

Camille La Fontaine, Stéphanie Belin, Laurent Barthe, Olga Roudenko, Valérie Briois

\section{To cite this version:}

Camille La Fontaine, Stéphanie Belin, Laurent Barthe, Olga Roudenko, Valérie Briois. ROCK: A Beamline Tailored for Catalysis and Energy-Related Materials from ms Time Resolution to $\mu \mathrm{m}$ Spatial Resolution. Synchrotron Radiation News, 2020, 33 (1), pp.20-25. 10.1080/08940886.2020.1701372. hal-03060220

\section{HAL Id: hal-03060220 https://hal.science/hal-03060220}

Submitted on 13 Dec 2020

HAL is a multi-disciplinary open access archive for the deposit and dissemination of scientific research documents, whether they are published or not. The documents may come from teaching and research institutions in France or abroad, or from public or private research centers.
L'archive ouverte pluridisciplinaire HAL, est destinée au dépôt et à la diffusion de documents scientifiques de niveau recherche, publiés ou non, émanant des établissements d'enseignement et de recherche français ou étrangers, des laboratoires publics ou privés. 


\title{
ROCK: a beamline tailored for catalysis and energy-related materials from ms time resolution to $\mu \mathrm{m}$ spatial resolution
}

\author{
Camille La Fontaine, Stéphanie Belin, Laurent Barthe, Olga Roudenko, Valérie Briois \\ Synchrotron SOLEIL, L'Orme des Merisiers, BP48, 91192 Gif-sur-Yvette, FRANCE
}

Assessing the structure-activity relationship in materials used as catalysts or for energy storage is a major challenge in order to provide efficiency, durability, clean processes and sustainable energy. Xray Absorption Spectroscopy (XAS) is a widely used technique to this aim, as it can provide structural and electronic information about the active elements of these materials. Many XAS beamlines have improved their performances to provide time-resolved information about evolving materials under real working conditions. To this end, Quick-EXAFS (QEXAFS) monochromators were installed at SOLEIL synchrotron on the SAMBA beamline in 2009 [1]. The excellent performances for operando studies of heterogeneous catalysts achieved on SAMBA [2] were very attractive for the scientific community, although the SAMBA's optical layout was optimized for its sagitally-focusing doublecrystal monochromator and could not offer more than $10^{11} \mathrm{ph} / \mathrm{s}$ flux at the best at the sample position when using the QEXAFS ones. Based on the experience gained on SAMBA, the construction of a new beamline dedicated and optimized for the QEXAFS monochromators was decided and granted in 2011 by the French Agence Nationale de la Recherche in the framework of the national "Investissements d'Avenir" program (ANR-10-EQX-45-01). In order to propose a beamline tailored for operando characterization of catalytic and energy related materials, both experts from the synchrotron world and from the scientific domains were gathered from the start of the project. The consortium, leaded by SOLEIL, involved three major French scientific actors of the heterogeneous catalysis and materials for the energy communities: the Unite de Catalyse et Chimie du Solide (UCCS, UMR 8181, Université de Lille), the Laboratoire de Réactivité de Surface (LRS, UMR 7197, Sorbonne Université) and the Réseau pour le Stockage Electrochimique de l'Energie (RS2E, FR 3459, CNRS). The new ROCK ("Rocking Optics for Chemical Kinetics") beamline opened to users in 2015 and is operated by three full-time SOLEIL's scientists, two of them working on heterogeneous catalysis and the third one on electrochemical energy storage, and one technician. In addition, the RS2E provides the beamline with one research engineer to support its experiments. Furthermore, thanks to the catalysis partners of the consortium but also public research actors like IFPen which supported the project of the ROCK beamline construction in 2011, PhD students are regularly granted by these external fundings to carry out their research program at the beamline with strong emphasis on methodological and technical developments, such as e.g. new catalytic reactors, which are then at the benefit of the whole scientific community. Through almost five years of operation, ROCK has reached state-of-the-art capabilities and gained strong expertise, offering dedicated sample environments, unique measurement methodologies involving multiple edge characterization and combination of spectroscopic techniques, and in-depth data analysis using chemometrics.

\section{General beamline description and performances}

The ROCK's optical layout has been described in details previously [3]. ROCK has been especially designed in order to fulfill the requirements of operando measurements: a high photon flux, a wide 
energy range from 4 to $43 \mathrm{keV}$ covering most of the elements of interest in catalysis, adequate time resolution and efficient harmonic rejection.

To maximize the photon flux collected from the bending magnet source $(1.72 \mathrm{~T}$, critical energy $=8.55$ $\mathrm{keV})$, a toroidal mirror is installed in the storage ring tunnel at $c a .10 .15 \mathrm{~m}$ from the source which ensures a $1.5 \mathrm{mrad}(\mathrm{H}) \times 0.22 \mathrm{mrad}(\mathrm{V})$ acceptance. According to its toroidal shape, this first mirror then focuses the beam horizontally at a fixed position inside the experimental hutch, allowing all the collected photons to propagate to the other optics. Thus, the photon flux achieved at the sample position is in the range $10^{11}$ to $10^{12} \mathrm{ph} / \mathrm{s}$ depending on the energy and for a machine current of 500 mA.

To cover permanently the 4 to $43 \mathrm{keV}$ energy range, Si(111) and Si(220) QEXAFS monochromators installed in series in the same vessel can be positioned alternately on the beam thanks to an in vacuum vertical translation stroke. These monochromators, described in [1], allow recording spectra with an oscillation frequency up to $20 \mathrm{~Hz}$, i.e. $25 \mathrm{~ms}$ per spectrum. The amplitude of the oscillations is adjustable from $0.3^{\circ}$ to nearly $4^{\circ}$ so that full EXAFS information can be collected whatever the absorption edge accessible at the beamline (from Ti to Ce for K edges). The originally Si(220) watercooled monochromator reduced the effective photon flux at the sample for high energy operation due to thermally-induced deformation on the first crystal larger than the intrinsic Darwin width. To improve the beamline performances at high-energy, a third QEXAFS monochromator with liquid nitrogen cooling has been designed by the SOLEIL's Design office and very recently installed. It is equipped with the Si(220) channel-cut crystal, while both water-cooled monochromators are still available with Si(111) crystals (Figure 1 ).

Efficient high-order harmonic rejection is ensured by setting the incidence of pink and monochromatic beams on two dedicated mirrors placed before and after the monochromators, respectively. The selection of one of the three coating strips $\left(\mathrm{B}_{4} \mathrm{C}, \mathrm{Pt}, \mathrm{Pd}\right)$ available on both mirrors offers three different cut-off energies at a single beam incidence without changing the optics and sample alignments. This design is of crucial importance for operando studies of catalysis and energy related materials which are getting more complex with compositions involving several metals as active centers. In addition to the easy adaptation of harmonic rejection properties, changing the energy is very fast even when the use of two different monochromators is needed, e.g. as required for the study of hydrodesulfurization CoMoS catalysts [4] where Si(111) is used for Co K-edge (7709 $\mathrm{eV})$ data collection and $\mathrm{Si}(220)$ for Mo K-edge $(20000 \mathrm{eV})$ data recording. An automated procedure manages the complete process: exchange of the monochromators (change of vertical position and main Bragg angle), selection of the correct strip on the mirrors for an efficient harmonic rejection, configuration of the acquisition chain (hardware and software), and optimization of the detection. The latter is achieved by using two different sets of 3 ionization chambers, each of them filled with a gas mixture ( $\mathrm{He}, \mathrm{N}_{2}, \mathrm{Ar}, \mathrm{Kr}$ ) adapted to the energy measured, and placed on top of each other and pneumatically actuated. The complete change in beamline configuration to jump from one edge to another one takes one minute in the worst case and $30 \mathrm{~s}$ generally. This "edge jumping" feature is highly demanded [4-7] as it provides multiple edge characterization on a material evolving under working conditions (temperature, pressure, or gas composition for catalysis, applied current or potential for energy related materials) in a single experiment. In this way, the experimentalist has a true depiction of the concomitant temporal evolutions of the different active centers subjected to exactly the same operating conditions. Figure 2 illustrates for instance the monitoring of industrial 
liquid sulfidation at 30 bar of a CoMoP catalyst using the edge jumping capability of the beamline. Alternate acquisitions at the $\mathrm{Co}$ and Mo $\mathrm{K}$ edges upon the 30 hours of catalyst activation were carried out in order to understand synergetic effect of sulfidation between Mo and $\mathrm{Co}$, as detailed in [4].

Beam size at the sample position can be varied in both horizontal and vertical planes. The horizontal size is changed by setting the complete experimental table (which supports the sample and the ionization chambers) at a continuum of positions inside the experimental hutch, from the focusing point of the first toroidal mirror to $c a .6 \mathrm{~m}$ upstream. The vertical size is controlled by the curvature of the cylindrically bent third mirror placed after the monochromators. Thus, the beam can be focused to $c a .380(\mathrm{H}) \times 130(\mathrm{~V}) \mu \mathrm{m}^{2} \mathrm{FWHM}$ or spread by one order of magnitude in both directions. As a result, the photons density impinging the sample can be adjusted by a factor 100 , which is of paramount importance for samples suffering from radiation damage, without altering the signal-tonoise ratio since the photon flux is preserved [3]. This versatility in beam size also allows accommodating different sample environments, such as large Lytle-type cells [2] or thin capillary reactors [7].

\section{Dedicated equipment for catalysis}

ROCK is designed for operando characterization of catalytic and energy related materials. To achieve this objective, the beamline provides dedicated facilities for heterogeneous catalysis. An overview is presented in Figure 3. Several reaction cells are available with complementary characteristics in terms of temperature and/or pressure of use. A general-purpose Lytle-type cell consists of a cavity of 1 to $6 \mathrm{~mm}$ of thickness which is filled with the catalyst powder (Figure $3(\mathrm{a})$ ). This cell, described in [2], allows atmospheric pressure experiments up to $600^{\circ} \mathrm{C}$. In addition, two capillary cells are also available. The first one uses a hot air gas blower from FMB Oxford and accommodates 1 to $3 \mathrm{~mm}$ diameter capillaries mounted in the hot air path and heated up to $900^{\circ} \mathrm{C}$. With adequate capillaries (e.g. $1.2 \mathrm{~mm}$ OD capillaries with $20 \mu \mathrm{m}$ wall thickness), this cell allows conducting experiments at pressures up to 20 bar e.g. for Fischer-Tropsch reaction. The second capillary cell available on ROCK has been recently designed by the beamline staff in order to provide a more controlled and stable temperature up to $1000^{\circ} \mathrm{C}$ (Figure $3(\mathrm{~b})$ ). The heating is ensured by a radiation heating element and is homogeneous over a length of $29 \mathrm{~mm}$. $115 \mathrm{~mm}$ long capillaries of diameters from 1.2 to $3 \mathrm{~mm}$ can be mounted. Finally, a new cell, based on the one described in [4], is currently under design and will be dedicated to high pressure/high temperature measurements inside vertically or horizontally mounted glassy carbon tubes $(O D=4.7 \mathrm{~mm}, I D=2.1 \mathrm{~mm}$ ) for gas and liquid phase experiments up to 30 bar and $600^{\circ} \mathrm{C}$.

Together with the reaction cells, the beamline offers the infrastructure for gas distribution. It includes a gas cabinet to safely dispose dangerous gases cylinders with efficient venting (Figure 3 (c)), and several distribution systems with mass flow controllers. Gas cabinet and distribution systems are located inside the experimental hutch, as close as possible from the catalytic reactor to reduce the length of tubing. The first distribution system allows mixing up to 5 different gases, offers the possibility to flow gases through a saturator to add a partial pressure of a liquid reactant (e.g. a mixture of water and an alcohol for steam reforming experiments), and can deliver gases at a pressure up to 20 bar (Figure 3 (c)). The second gas distribution system is intended to subject catalysts to periodic changes of gas composition for modulated excitation experiments [8]. It 
accommodates fast switching valves to exchange alternately between up to four different gases with automated actuation. A last gas distribution system is dedicated to experiments involving dihydrogen sulfide. To ensure a high level of safety, detectors are permanently installed in the experimental hutch for $\mathrm{CO}, \mathrm{NO}, \mathrm{H}_{2} \mathrm{~S}$, and $\mathrm{H}_{2}$ with possible additional detectors provided on purpose by SOLEIL Safety group for other specific gases.

In order to provide information on the activity and selectivity of the catalysts which is mandatory for a true operando experiment, online gas analysis is achieved by a mass spectrometer (Cirrus, MKS, 1$200 \mathrm{amu}$, Figure 3 (c)). In addition, gas monitoring can also be performed thanks to Raman spectroscopy using Raman gas probes [4, 9] available on ROCK. The Raman probes can be installed at any position in the gas stream even when operating at high pressure. This is an advantage over the mass spectrometer which is placed at the gas outlet at atmospheric pressure and leads to a strongly delayed response.

Recently, a new cell suitable for electrocatalysis was designed for the purpose of a user project related to the electrochemical reduction of $\mathrm{CO}_{2}$. This home-made cell is a gas-tight two compartment electrochemical cell (Figure 3 (d)). Its use requires the detection of the signal in fluorescence mode using a Passivated Implanted Planar Silicon detector with $3000 \mathrm{~mm}^{2}$ active area. The electrochemistry is controlled and monitored thanks to a multi-channel potentiostat (VMP3, BioLogic, Figure 3 (e)) equipped with an external booster (VMP3B, Bio-Logic) to deliver up to $2 \mathrm{~A}$ and $20 \mathrm{~V}$. This potentiostat is also used for the operando studies of electrode materials for Li-ion or Naion batteries conducted with a dedicated electrochemical cell [10].

\section{Combination of techniques}

In order to provide a deeper insight into the system under investigation, using complementary techniques in a combined way is often very valuable. ROCK scientists have a strong background in combining XAS with other spectroscopies [11]. Such coupled experiments are regularly operated on ROCK where UV-Vis (Cary 50, Varian) and Raman (Kosi RXN1 $785 \mathrm{~nm}$ and RXN1 $532 \mathrm{~nm}$, Kaiser) spectrometers are available. UV-Vis spectroscopy can provide important information about e.g. the nucleation and growth of nanoparticles in solution [12], while QEXAFS spectra are collected to monitor the relative proportion of precursors and nanoparticles in solution.

Thanks to optical fibers and long working-distance objectives, Raman spectra can be collected on the three cells available for catalysis (directly through the quartz capillaries or through a $25 \mu \mathrm{m}$ mica window for the Lytle-type cell). Raman spectroscopy can provide complementary information about the sample's structure [13-14] and also about surface carbonaceous deposits [15-16]. As an example, the combination of QEXAFS and Raman spectroscopy has recently been applied to monitor the deactivation of $\mathrm{Co} / \mathrm{Al}_{2} \mathrm{O}_{3}$ catalyst during ethanol steam reforming (ESR) carried out at $500^{\circ} \mathrm{C}$ under a He flow saturated with $\mathrm{C}_{2} \mathrm{H}_{5} \mathrm{OH} / \mathrm{H}_{2} \mathrm{O}$ in the ratio $1: 6$, and, its subsequent regeneration under oxidative atmosphere [15]. The set-up used for the combination of both techniques is shown in Figure 4 (a). After 90 min of ESR, deactivation has been observed by Mass Spectrometry used to monitor the ethanol conversion into $\mathrm{H}_{2}$. The deactivation has been related by Raman spectroscopy to the formation of coke at the catalyst surface. Introducing $\mathrm{O}_{2}$ to the gas feed allows the burning of the coke and after $\sim 20$ min of this treatment, a decrease of the intensity of the Raman lines characteristic 
for coke deposits ( $D$ line at $1340 \mathrm{~cm}^{-1}$ and $\mathrm{G}$ line at $1585 \mathrm{~cm}^{-1}$ ) is observed (Figure 4 (c)) together with an increase of the intensity of the white line of the QEXAFS spectra (Figure 4 (b)) pointing out the onset of reoxidation of the $\mathrm{Co}(0)$ active species $(55 \% \mathrm{Co}(0)$ at $\mathrm{t}=30 \mathrm{~min}$ against $88 \%$ at $\mathrm{t}=0 \mathrm{~min}$ ). The combination of Raman spectroscopy and XAS was unique for controlling the regeneration since $\mathrm{O}_{2}$ flow was stopped as soon as no more lines of coke were detected by Raman. After coke removal the activity came back to the level before deactivation promoted by the $\mathrm{H}_{2}$ produced during ESR through a self-reactivation process of the catalyst [13].

\section{Improving skills for data treatment}

Performing a time-resolved QEXAFS experiment with time resolution ranging from 25 to $250 \mathrm{~ms}$ per spectrum yields to hundreds or even thousands of spectra in a couple of minutes. Such high time frame is mandatory in catalysis to unravel the presence of intermediate species. However, rapidly we were aware that the users can be lost among the huge amount of spectra collected and needs to use adapted tools in order to extract the relevant information contained in their thousands of spectra without analyzing them one by one. With the advent of chemometrics, dealing with such huge datasets becomes accessible. ROCK scientists have been very active in the past ten years for the use of Multivariate Curve Resolution with Alternating Least Squares fitting (MCR-ALS) for XAS data. The methodology available on ROCK is now well established and involves (i) Principal Component Analysis - Singular Value Decomposition (PCA-SVD) to identify the number of components needed to fully represent the variance of the dataset, and (ii) MCR-ALS to extract, on the basis of the bilinear decomposition of the dataset obeying to the Beer-Lambert law, spectra of the pure components involved in the reaction together with their concentration profiles [4, 14-18]. An example of speciation so-obtained by MCR-ALS for the Mo K edge data presented in Figure 2 (a) is shown in Figure 2 (c). For this catalyst [4], liquid sulfidation is first described by a transformation of a first oxydic species (Mo-Ox1) into two intermediate oxidic (Mo-Ox2) and oxysulfide $\left(\mathrm{MoO}_{x} \mathrm{~S}_{\mathrm{y}}\right)$ species, the latter being massively produced above $233^{\circ} \mathrm{C}$ when dimethyldisulfide molecules are decomposed into $\mathrm{H}_{2} \mathrm{~S}$. At higher temperatures, fully sulfided species $\mathrm{MoS}_{3}$ and $\mathrm{MoS}_{2}$ are formed. MCR-ALS is performed using the MCR-ALS $2.0 \mathrm{GUI}$ developed for Matlab environment by the group of Roma Tauler [19]. This Matlab GUI requires as input a matrix containing XAS spectra which are first properly calibrated in energy and normalized. This first step is realized thanks to the normal_gui python interface developed at SOLEIL, which allows processing hundreds of spectra in only few seconds [4]. Regular tutorials are provided to the users in order to practice by themselves this multivariate analysis which allows resolving complex mixtures involving multiple intermediate species which could not be identified otherwise.

\section{Towards hyperspectral imaging}

Adding spatial information to time-resolved data would be very valuable to probe heterogeneities in the investigated systems, such as speciation gradients along catalytic beds or on shaped catalysts. Such development is under progress on ROCK by implementing a pixelated detector (ORCA-Flash4.0 Digital CMOS camera, Hamamatsu) to collect the visible light emitted by a thin scintillator (e.g. $70 \mu \mathrm{m}$ of LuAG:Ce) in the path of X-rays transmitted by the sample. Images are collected with an exposure time of $5 \mathrm{~ms}$ and repeated during the oscillation of the QEXAFS monochromator whose velocity is decreased to $0.1 \mathrm{~Hz}$. As a result, XAS spectra can be reconstructed on each pixel, or on binned pixels 
to improve the signal if necessary. Depending on the objective mounted on the camera (magnification X10 or X5), the pixel size and maximum field of view range from $0.65 \mu \mathrm{m}$ to $1.3 \mu \mathrm{m}$ and $1.3 \mathrm{~mm}$ to $2.6 \mathrm{~mm}$, respectively, with a resolution of $c a .5 \mu \mathrm{m}$. The first experiments carried out have demonstrated the potentiality of this approach to gain spatial XAS information. Then, using MCR-ALS analysis of the reconstructed spectra on each pixel, it was possible to assess the spatial speciation of a sample made of $\mathrm{CuO}$ and $\mathrm{Cu}_{2} \mathrm{~S}$ as shown in Figure 5.

Very recently, a test experiment combining both spatial and time resolutions allowed monitoring the reduction at $500^{\circ} \mathrm{C}$ of a supported Cu catalyst occurring in less than one minute inside a capillary cell, while showing different speciation along the catalytic bed. This result confirms the great potentiality of hyperspectral imaging and the capabilities of ROCK to achieve such challenging development.

\section{Conclusion}

The ROCK beamline has been tailored for in situ/operando investigations of a wide range of materials (catalysts, electrocatalysts, battery electrodes, nucleating nanoparticles, etc.). The beamline offers home-made cells for different applications in temperature and pressure. Most of them are designed for allowing combination of techniques such as Raman spectroscopy providing valuable complementary information for better understanding complex mechanisms involved at different stages of the catalyst life span. The edge jumping capability available at the beamline is largely requested by the users with about $50 \%$ of proposals which require characterizations of two or more elements in the same materials upon working conditions. The bottleneck of huge amount of data analysis has been overcome by the implementation at the beamline of automated normalization and energy calibration skills allowing the further use of multivariate data analysis based on the MCR-ALS approach. On-line MCR-ALS analysis can be performed at the beamline which is of paramount importance for allowing the users to interactively optimize the experiment under progress.

\section{Acknowledgement}

The beamline construction and operation (2011-2019) were supported by a public grant overseen by the French National Research Agency (ANR) as a part of the "Investissements d'Avenir" (ref: ANR-10EQPX-45).

\section{References}

1. E. Fonda et al., Journal of Synchrotron Radiation 19, 417-424 (2012).

2. C. La Fontaine et al., Catalysis Today 205, 148-158 (2013).

3. V. Briois et al., Journal of Physics: Conference Series 712, 012149 (2016).

4. C. Lesage et al., Catalysis Today 336, 63-73 (2019).

5 M. S. Nikulshina et al., Catalysis Science and Technology 8, 5557-5572 (2018).

6. J. E. S. van der Hoeven et al., ACS Nano 12, 8467-8476 (2018).

7. P. Sprenger et al., ACS Catalysis 8, 6462-6475 (2018).

8. C. F. J. König et al., Journal of Physical Chemistry C 116, 19857-19866 (2012).

9. A. Rochet et al., Catalysis Today 205, 94-100 (2013). 
10. J. B. Leriche et al., Journal of The Electrochemical Society 157(5), A606-A610 (2010).

11. V. Briois et al., Physica Scripta T115, 38-44 (2005).

12. B. L. Caetano et al., Journal of Physical Chemistry C 121, 886-895 (2017).

13. C. La Fontaine et al., Phase Transitions 84(8), 700-713 (2011).

14. A . Rochet et al., Journal of Physical Chemistry C 121, 18544-18556 (2017).

15. A. R. Passos et al., Catalysis Science \& Technology 8(24), 6297-6301 (2018).

16. A. R. Passos et al., Catalysis Today 336, 122-130 (2019).

17. W.H. Cassinelli et al., Catalysis Today 229, 114-122 (2014).

18. A. Rochet et al., Comptes Rendus Chimie 19, 1337-1351 (2016).

19. J. Jaumot et al., Chemometrics and Intelligent Laboratory Systems 140, 1-12 (2015). 


\section{Figure Captions}

Figure 1: The three QEXAFS monochromators of ROCK designed and assembled by the SOLEIL's Design office.

Figure 2: Monitoring of industrial liquid sulfidation at 30 bar of a CoMoP catalyst using the edge jumping capability of the beamline consisting of alternate acquisitions at the (a) Mo (each spectrum averaged over $35 \mathrm{~s}$ ) and (b) Co (each spectrum averaged over $305 \mathrm{~s}$ ) K edges (with $35 \mathrm{~s}$ for exchanging the monochromators), with (c) the evolution of the concentration profiles of Mo-based species determined by MCR-ALS.

Figure 3: Overview of some of the facilities for catalysis available on ROCK.

Figure 4: Combination of QEXAFS and Raman spectroscopy to monitor the regeneration of a $\mathrm{Co} / \mathrm{Al}_{2} \mathrm{O}_{3}$ catalyst during ethanol steam reforming. (a) Picture of the set-up used for the experiment, (b) QEXAFS spectra at Co K-edge and (c) Raman spectra recorded during the regeneration.

Figure 5: First hyperspectral imaging result gained on pellet made of a mixture of $\mathrm{CuO}$ and $\mathrm{Cu}_{2} \mathrm{~S}$ powders (a): pure component spectra obtained by MCR-ALS of the dataset (b) and their corresponding spatial distributions over a $1.8 \times 0.9 \mathrm{~mm}$ field of view (c). 
Figure 1

$1 \mathrm{LN}_{2}$-cooled monochromator

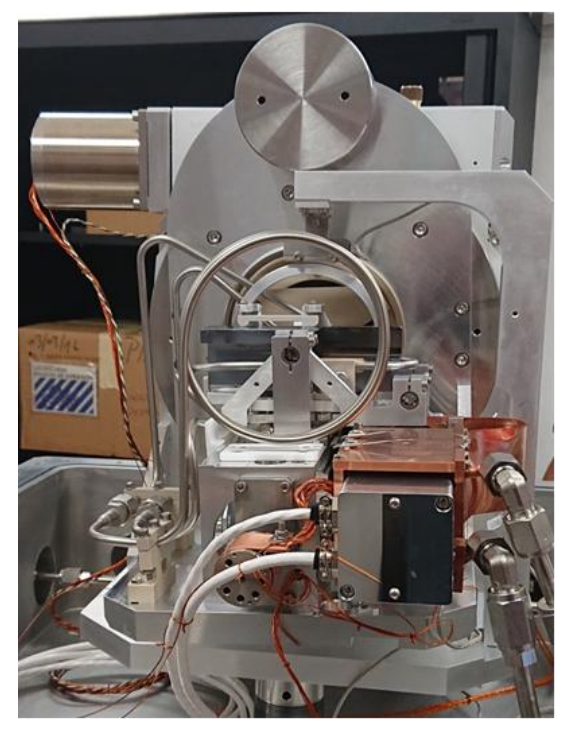

$\mathrm{Si}(220)$

6 - $43 \mathrm{keV}$
2 water-cooled monochromators

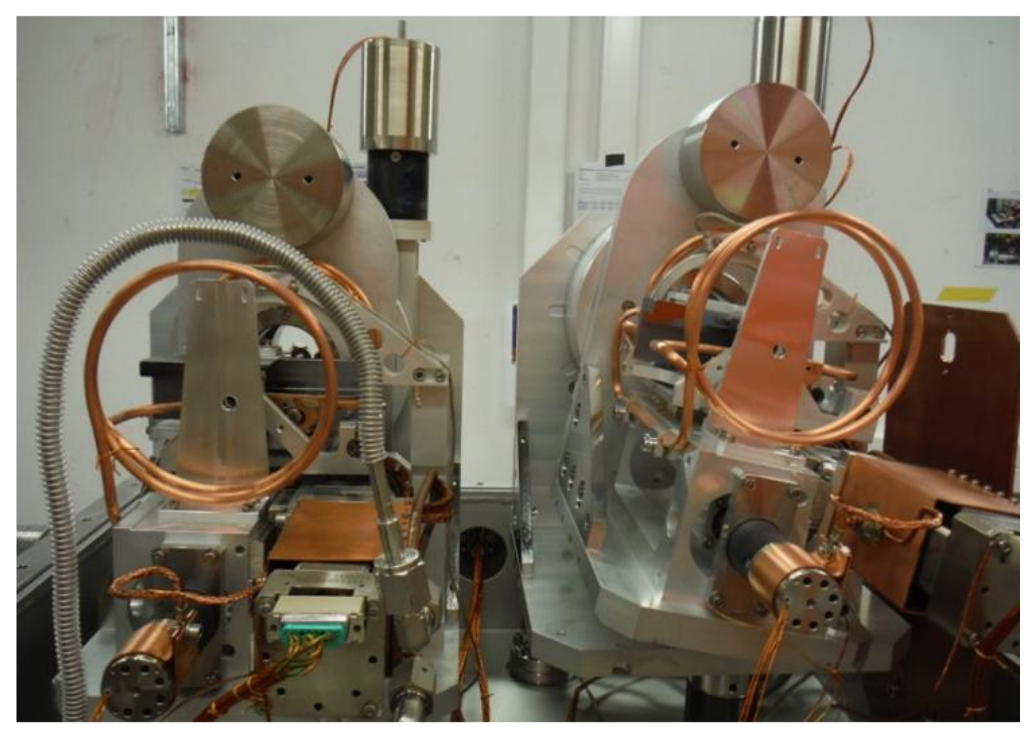

$\mathrm{Si}(111)$

$\mathrm{Si}(111)$

4 - $26 \mathrm{keV}$ 
Figure 2

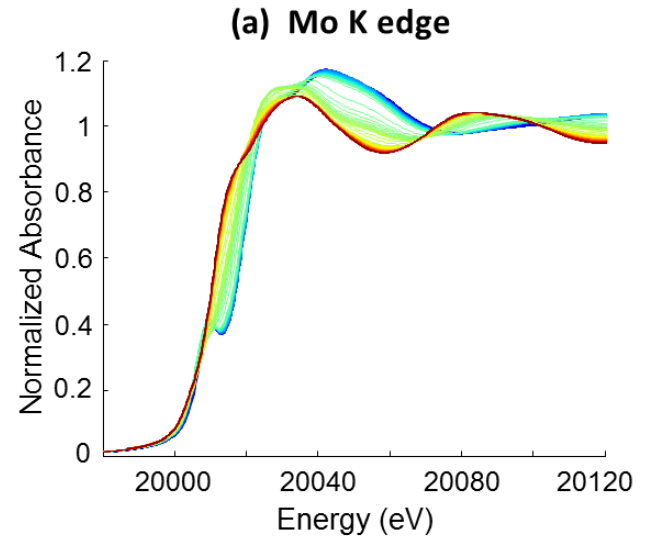

(b) Co K edge

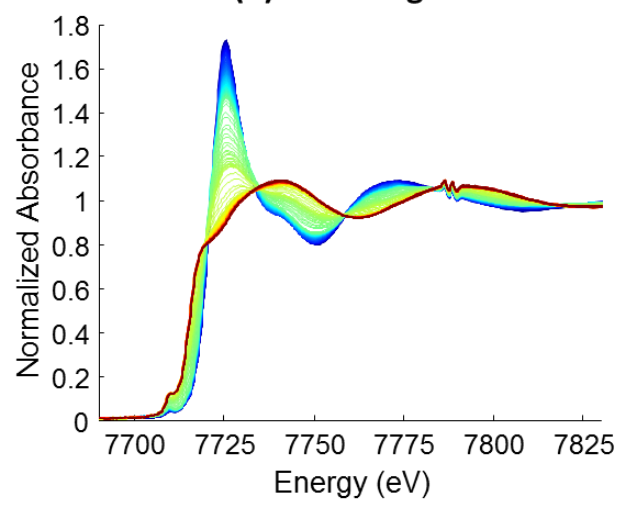

(c) Mo speciation

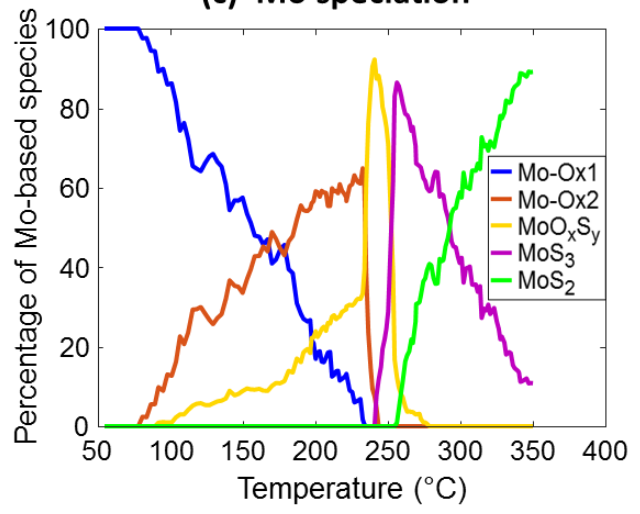


Figure 3

(a) Lytle-type cells

1 bar, $R T-600^{\circ} \mathrm{C}$

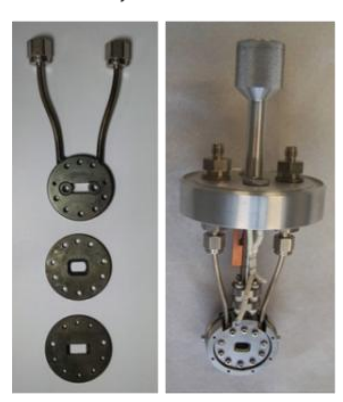

(d) Electrocatalytic cell

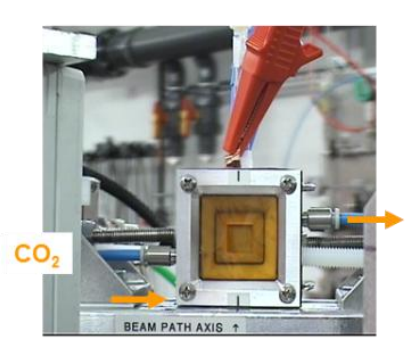

(b) Capillary cells

$20 \mathrm{bar}, R T-1000^{\circ} \mathrm{C}$

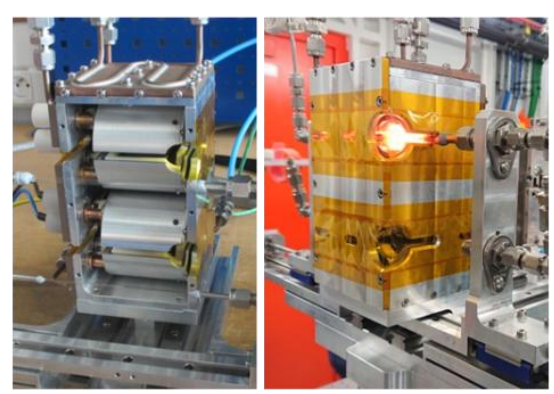

(e) Multi-channel Potentiostat

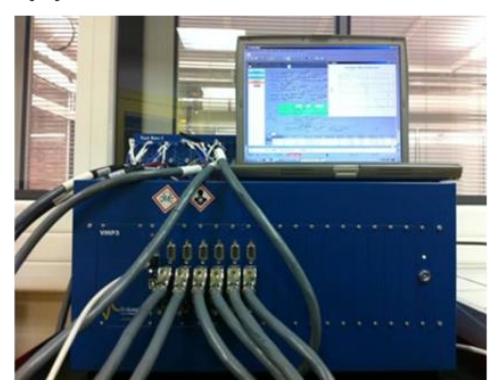

(c) Gas delivery system and analysis

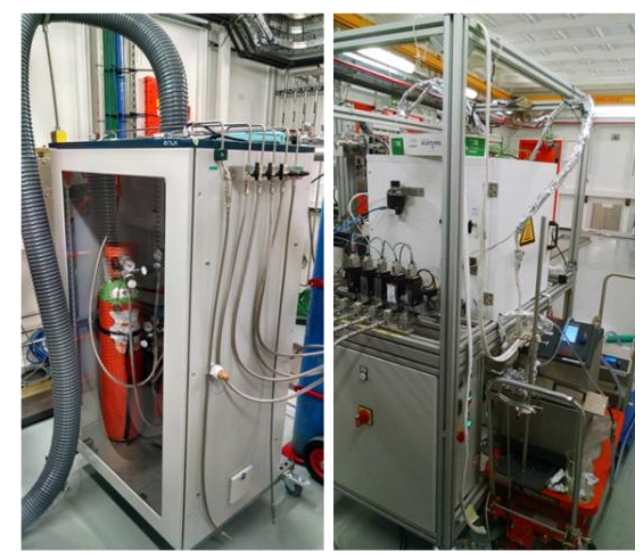

- 6 Mass Flow Controllers

- 1-200 amu. MS (Cirrus, MKS)

- Raman spectrometer

- Raman gas probe (532 nm laser, $175-4350 \mathrm{~cm}^{-1}$ )

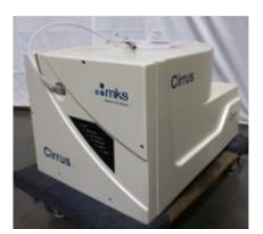


Figure 4

(a) Set-Up for Combination

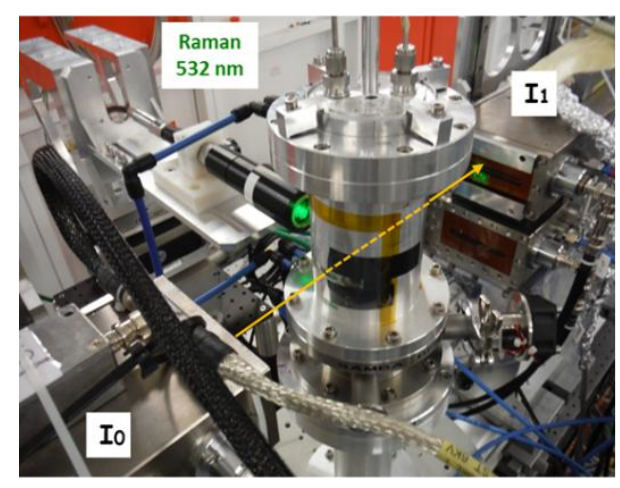

(b) QEXAFS

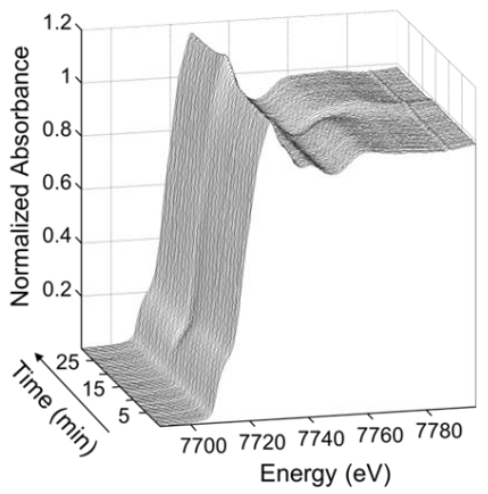

(c) Raman

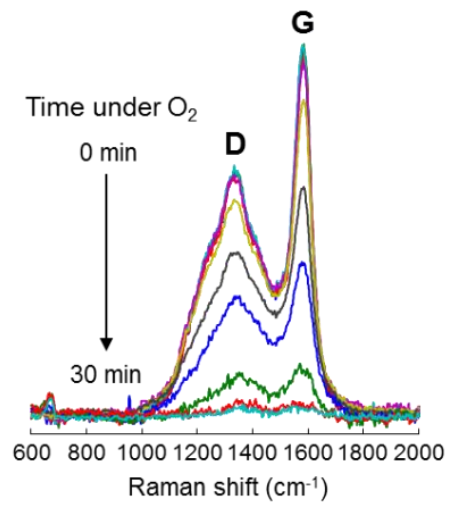


Figure 5

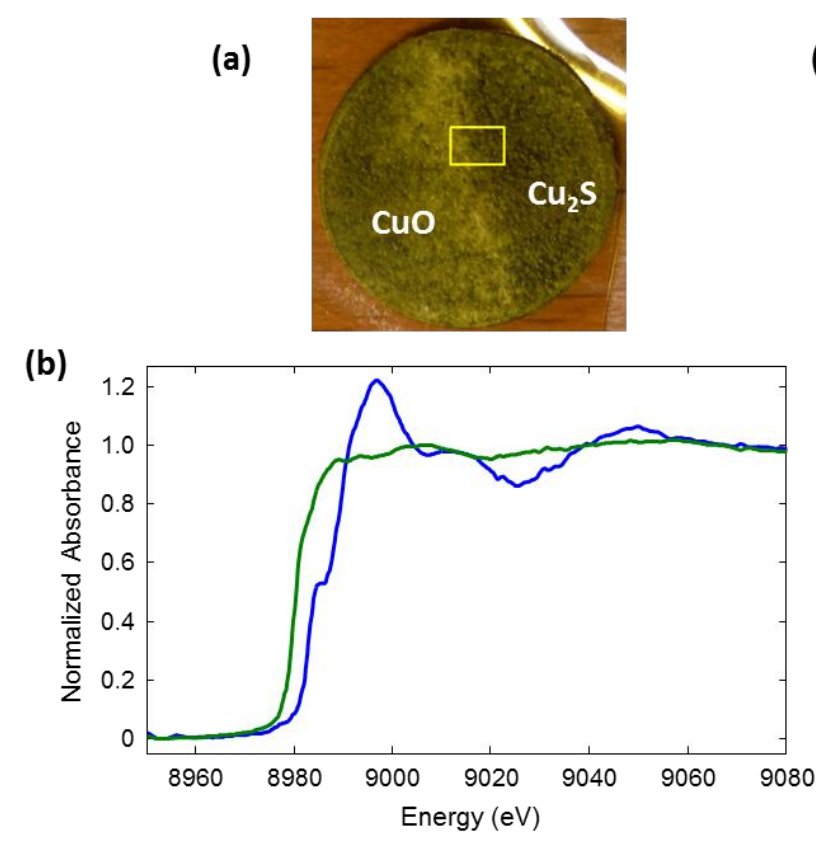

(c) Spatial distribution of
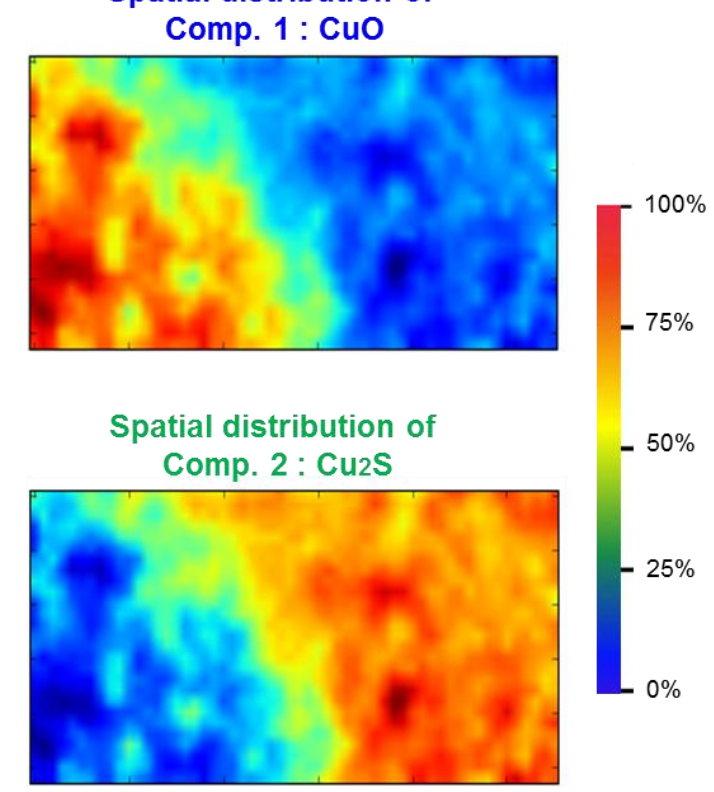